\title{
Nitro-Aldol approach for commercial manufacturing of Fenspiride Hydrochloride
}

Chinmoy Pramanik ${ }^{\dagger *}$, Kiran Bapat ${ }^{\dagger \S}$, Pradip Patil ${ }^{\dagger}$, Sandeep Kotharkar ${ }^{\dagger}$, Yogesh More ${ }^{\dagger}$, Dinkar Gotrane ${ }^{\dagger}$, Sudhir P. Chaskar ${ }^{\dagger \S}$, Ulhas Mahajan ${ }^{\dagger}$ Narendra K. Tripathy ${ }^{\dagger}$.

$†$ †PI R\&D Centre, Emcure Pharmaceuticals Ltd., ITBT Park, Phase-II, MIDC, Hinjewadi, Pune-411057, India

$\S$ Department of Chemistry, Sabitribai Phule Pune University, Ganeshkhind, Pune, Maharashtra, 411007

*Fax: +91-20-39821445.E-mail: chinmoy.pramanik@emcure.co.in 


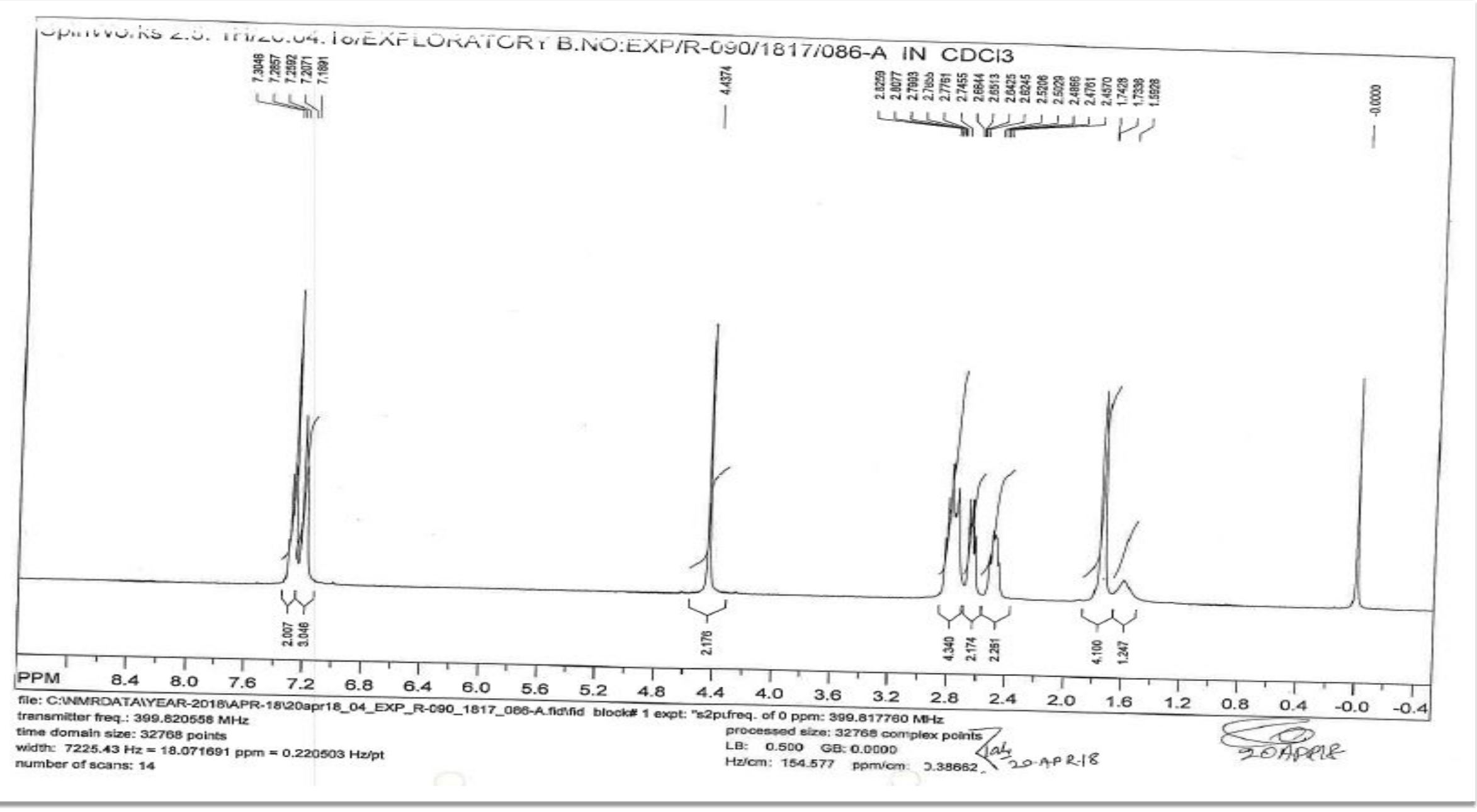

${ }^{1} \mathrm{H}$ NMR of compound 13 in $\mathrm{CDCl}_{3}$ 


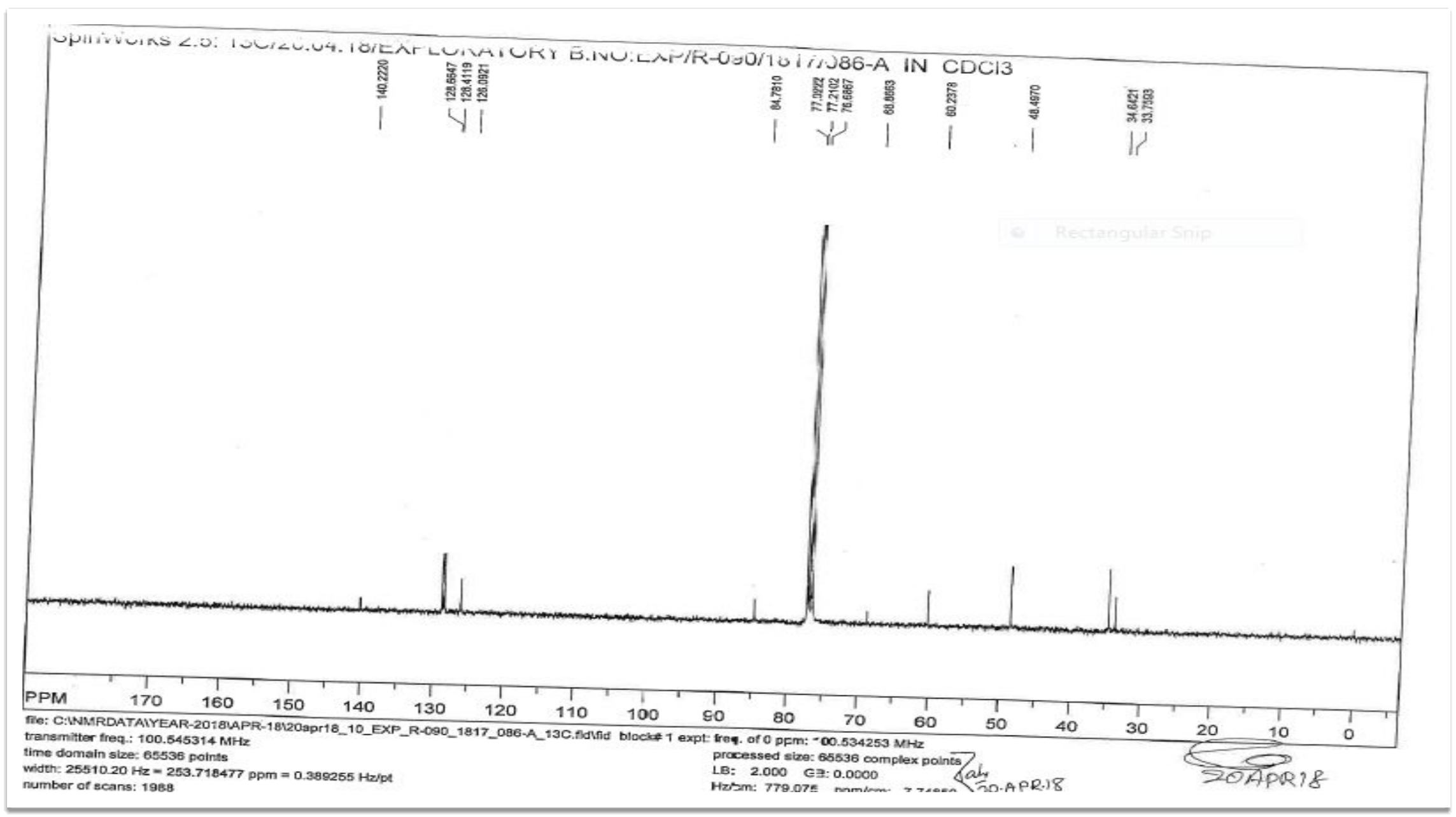

${ }^{13} \mathrm{C}$ NMR of compound 13 in $\mathrm{CDCl}_{3}$ 
Workstationi 4000 CTRAP
Operatort Singh, Dbhay

ENCURE PLRPMLEUTICALS LTD, ICMS LAB, API, HTRJNADI

Acq. Time: $15: 17$
Aca. Date: Setur

Instrument $\mathrm{ID:}-\mathrm{HI} / 2 \mathrm{AR} / 236$

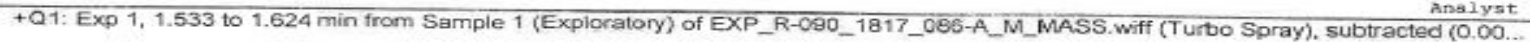

$\operatorname{Max} 4.9 \mathrm{eg}$

4. 906

4.8e6

4. $4 \mathrm{ee} 6$

$4.2 e 6$ -

3. 006

3.6e6

$3.4 e 6$

$3.0 \mathrm{e} 6$

뭉

2. 8 e6

2. 4 e6

$2.2 \mathrm{ec}$

2.006
$1.8 e 6$

1. $6 \mathrm{ee}$

1. $4 \mathrm{eb}$

1. $2 \mathrm{ec}$

8.005

6.0es

4.0es

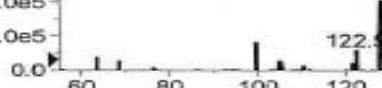

60
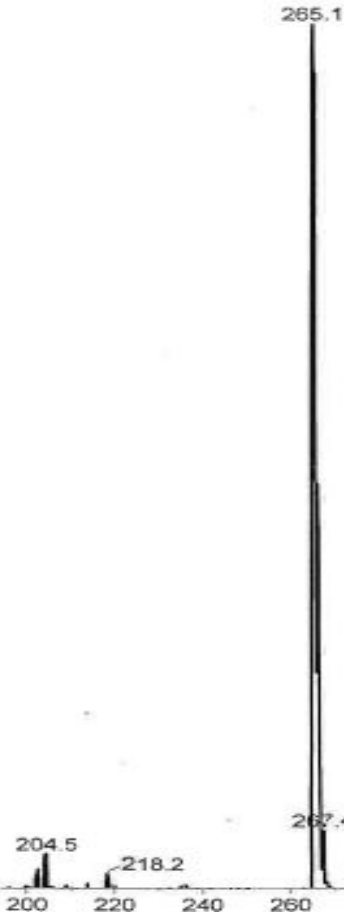

260

280300

Page 1 of 1

$380 \quad 400 \quad 420 \quad 440 \quad 460 \quad 480 \quad 500$

Printing Fine: $09: 13: 57$
Printing Date: 23 ppril 2010

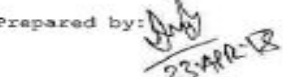

checked घy :

MASS of compound 13 


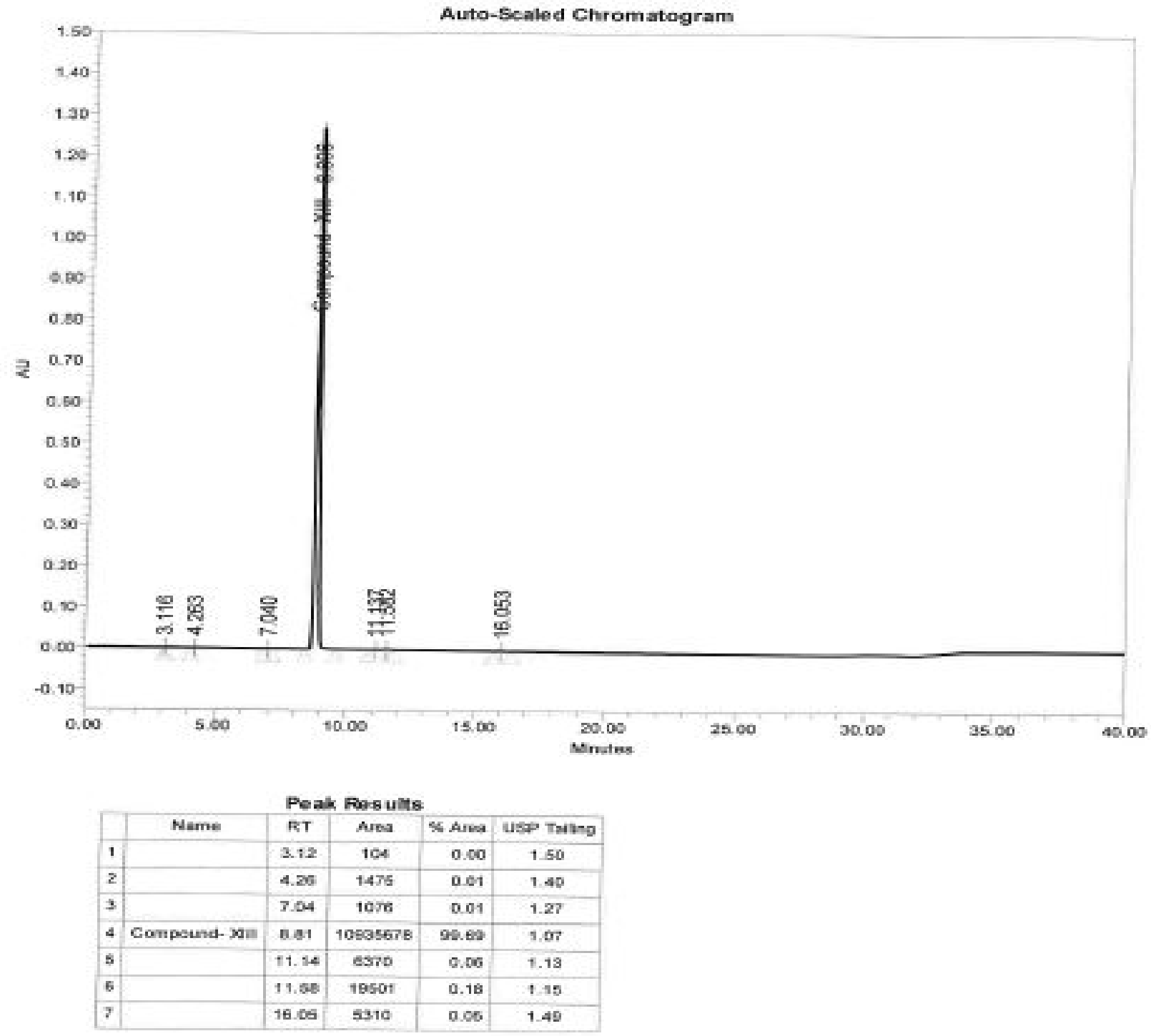

HPLC Chromatograms of compound 13 


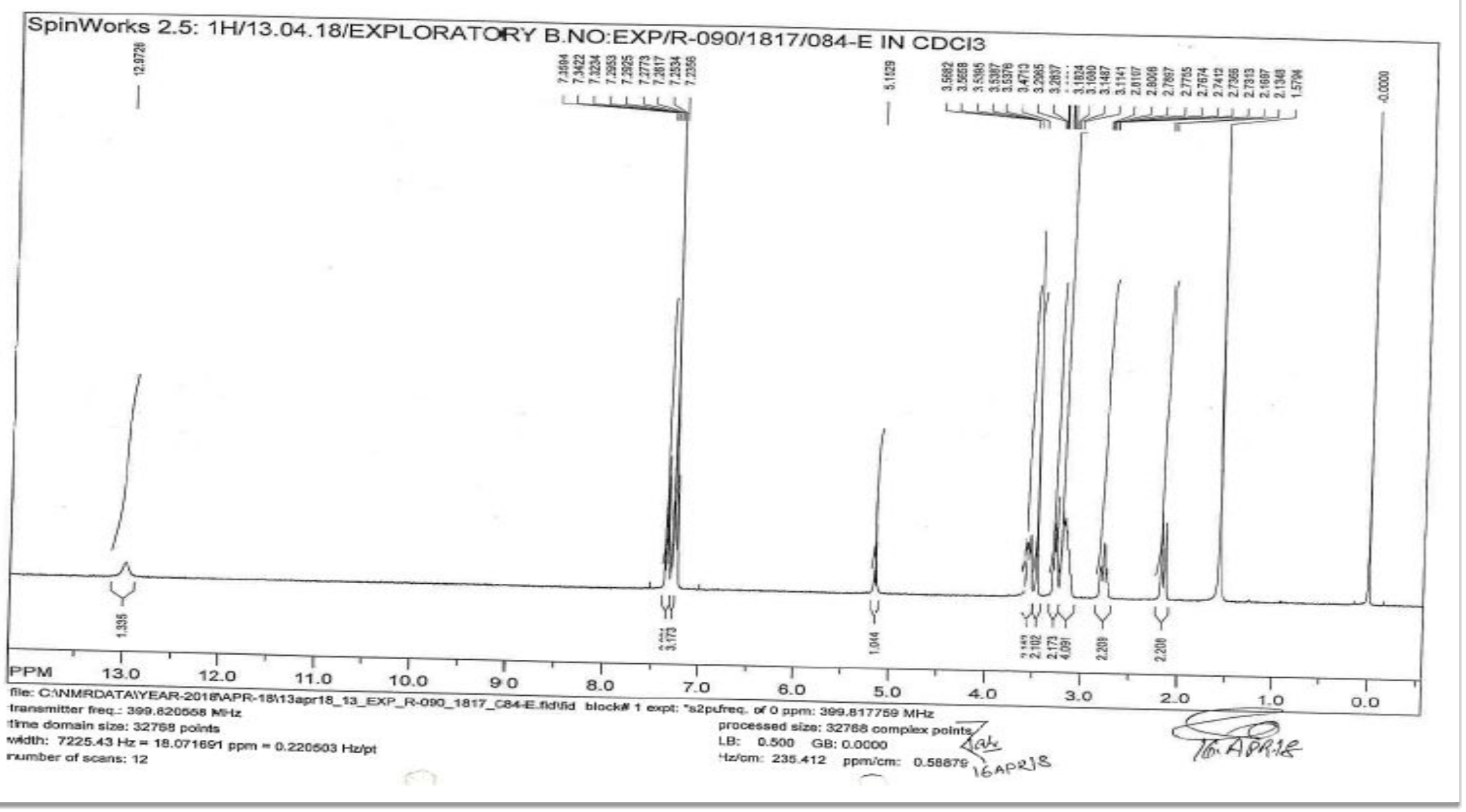

${ }^{1} \mathrm{H}$ NMR of compound 1 in $\mathrm{CDCl}_{3}$ 


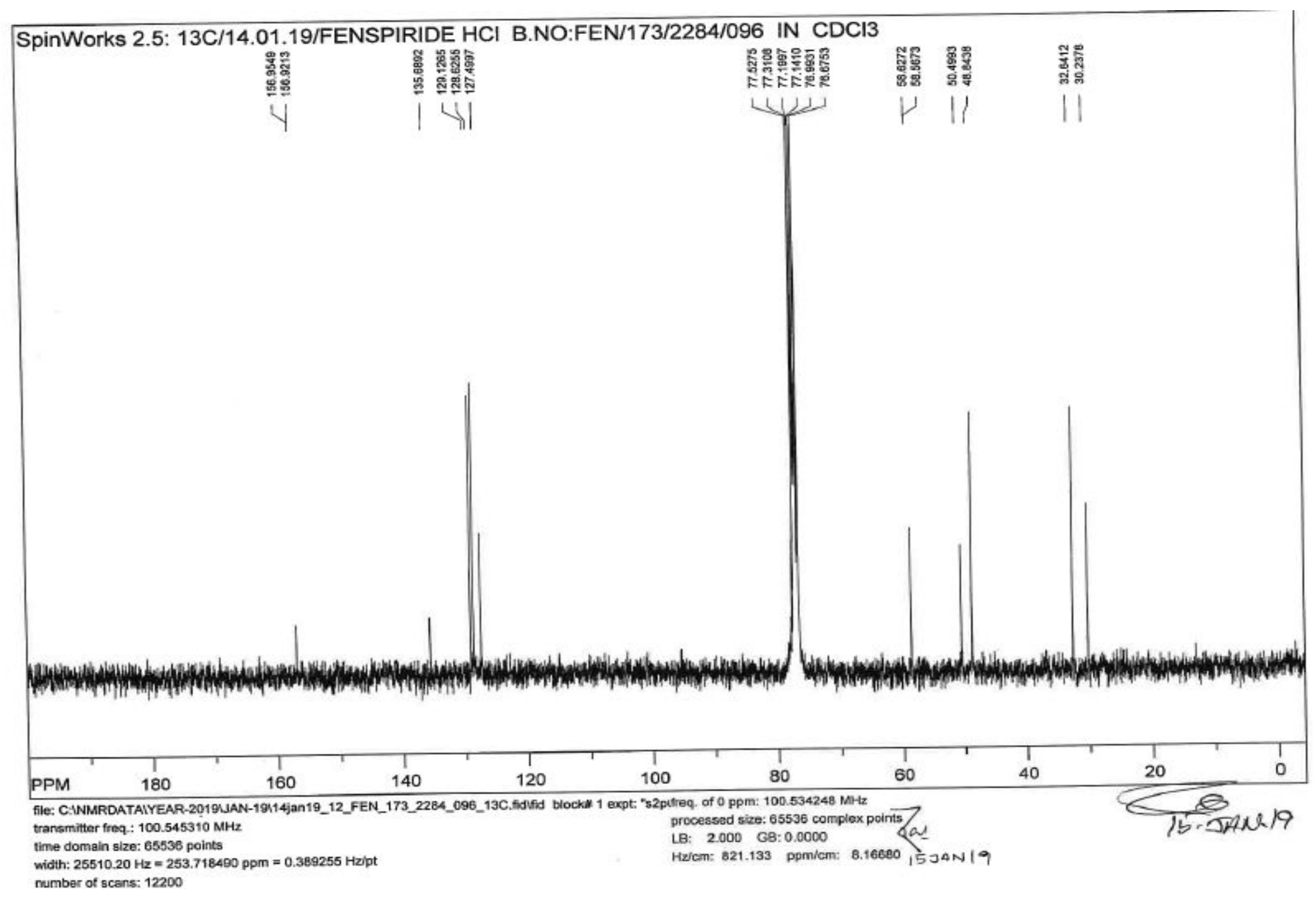

${ }^{13} \mathrm{C}$ NMR of compound 1 in $\mathrm{CDCl}_{3}$ 


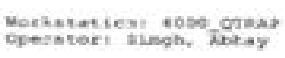

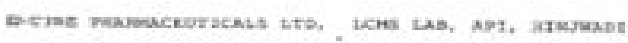

Ace. Tine: 13:33

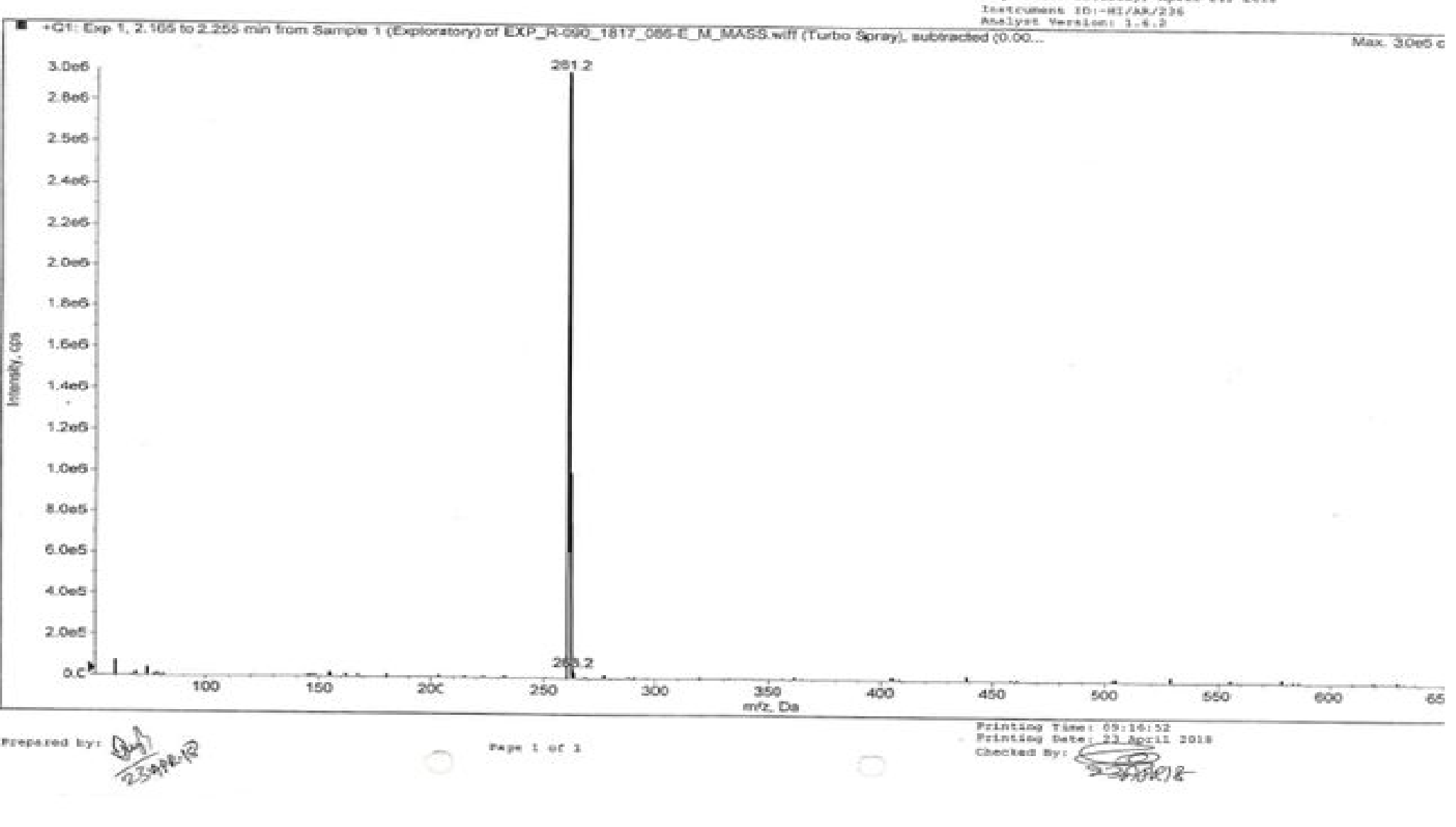

MASS of compound 1 
$<$ Chromatogram $>$

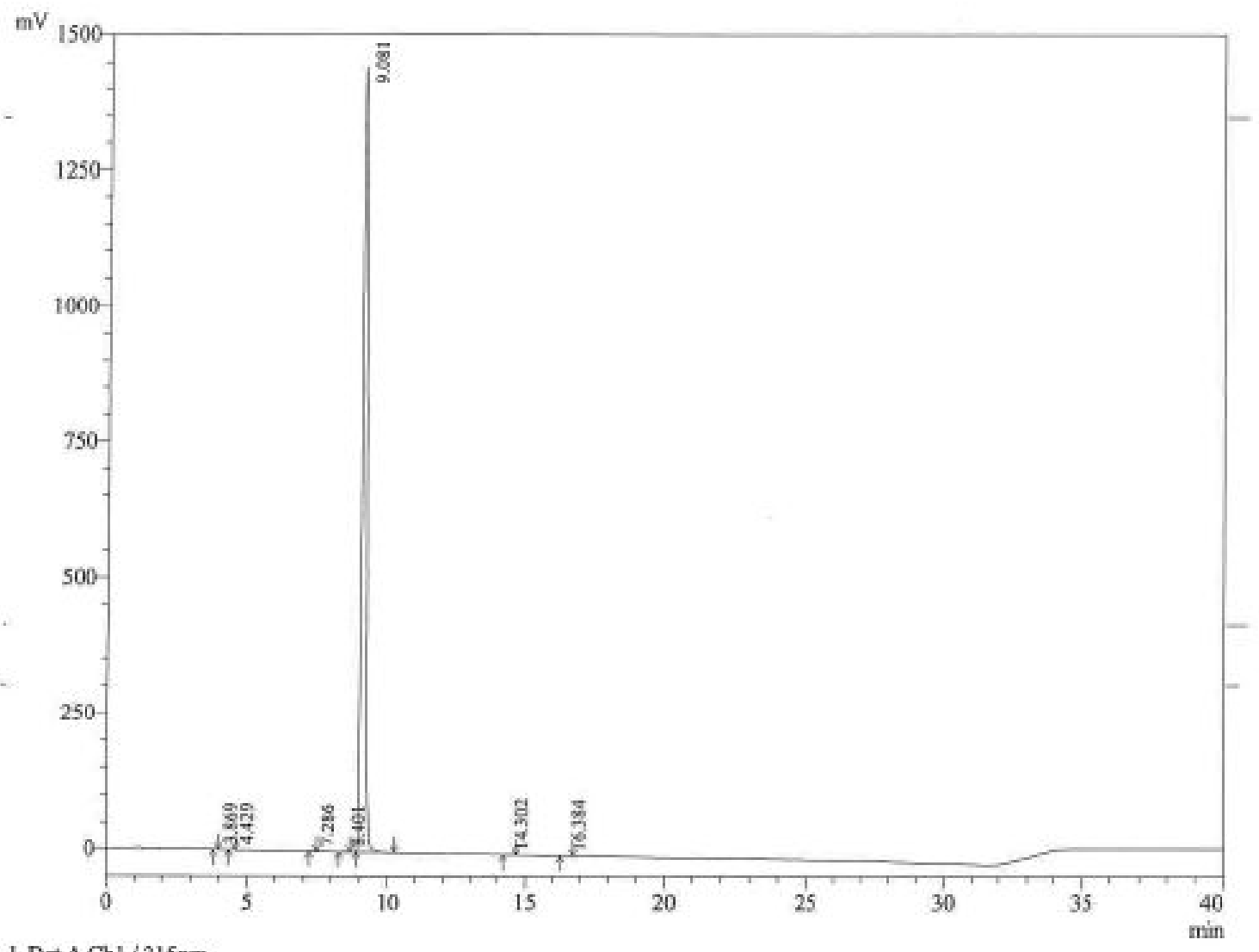

I Det.A Chl / 215nm

PeakTable

\begin{tabular}{|c|c|c|c|c|c|}
\hline Feakip & $N_{2 m e}$ & Res Time & Area & Area $\%$ & RET \\
\hline 1 & & 387 & 794 & 000 & 0.43 \\
\hline 2 & & 4.43 & 2015 & 0.01 & 0.49 \\
\hline 3 & & 7.29 & 2293 & 0.01 & 0.80 \\
\hline 4 & & 8.40 & 3449 & 0.02 & 0.93 \\
\hline 5 & Fenspinide (1) & 9.08 & 15866209 & 99.92 & 1.00 \\
\hline 6 & & 14.30 & 2886 & 0002 & 1.57 \\
\hline 7 & & 1638 & 1703 & 0.01 & 1.80 \\
\hline Tocal & & & 15874349 & 10000 & \\
\hline
\end{tabular}

HPLC Chromatograms of compound 1 